Résumés des conférences et travaux

\title{
Du Gandhāra à Socotra
}

Nouvelles découvertes de manuscrits indiens et d'inscriptions

Ingo Strauch

\section{OpenEdition \\ Journals}

Édition électronique

URL : https://journals.openedition.org/ashp/1208

DOI : 10.4000/ashp.1208

ISSN : 1969-6310

Éditeur

Publications de l'École Pratique des Hautes Études

Édition imprimée

Date de publication : 1 octobre 2011

Pagination : 240-243

ISSN : 0766-0677

Référence électronique

Ingo Strauch, «Du Gandhāra à Socotra », Annuaire de l'École pratique des hautes études (EPHE), Section des sciences historiques et philologiques [En ligne], 142 | 2011, mis en ligne le 27 juillet 2011, consulté le 06 juillet 2021. URL : http://journals.openedition.org/ashp/1208; DOI : https://doi.org/10.4000/ashp. 1208 


\title{
DU GANDHĀRA À SOCOTRA \\ NOUVELLES DÉCOUVERTES DE MANUSCRITS \\ INDIENS ET D'INSCRIPTIONS
}

\author{
Conférences de M. Ingo Strauch, \\ Freie Universität Berlin, \\ maître de conférences invité
}

Le cycle de conférences a présenté plusieurs découvertes nouvelles dans les domaines de l'épigraphie indienne et des anciens manuscrits bouddhiques. L'objectif principal était d'évaluer la portée de ces trouvailles par rapport aux données déjà connues de l'histoire religieuse et culturelle de l'Inde.

Nos connaissances sur la littérature bouddhique ancienne ont considérablement évolué au cours de la dernière décennie. Cela est dû principalement à un grand nombre de trouvailles spectaculaires de manuscrits dans une zone qui est partagée aujourd'hui entre le Pakistan et l'Afghanistan, et qui correspond plus ou moins à l'ancien Gandhāra. Les plus anciens d'entre eux se situent dans une période qui s'étend du $\mathrm{I}^{\mathrm{er}}$ siècle avant notre ère jusqu' $\mathrm{au} \mathrm{III}^{\mathrm{e}}$ siècle de notre ère. Par conséquent, ils ne constituent pas seulement les tout premiers manuscrits qui préservent des textes bouddhiques, mais même les premiers manuscrits, au sens strict, du monde indien, immédiatement après les inscriptions d'Aśoka (milieu du $\mathrm{III}^{\mathrm{e}}$ siècle avant notre ère). Ces documents sont écrits dans l'écriture propre à l'aire du Nord-Ouest, à savoir la kharoșthī, écriture d'origine araméenne, qui n'était pas seulement employée au Gandhāra proprement dit, mais aussi dans des régions limitrophes et jusqu'en Asie centrale, dans une large période comprise entre Aśoka et le $\mathrm{III}^{\mathrm{e}}$ siècle de notre ère dans son aire d'origine, à savoir le " Grand Gandhāra », et même jusqu'aux VI ${ }^{\mathrm{e}}-\mathrm{VII}^{\mathrm{e}}$ siècles de notre ère dans des communautés isolées du bassin du Tarim (actuel Xinjiang, Ouest de la Chine). Cependant, la grande masse de la documentation en kharoșthī appartient à la période principale de son emploi, c'est-à-dire jusqu'au $\mathrm{III}^{\mathrm{e}}$ siècle de notre ère. Bien qu'il y ait eu quelques tentatives d'employer la kharoșțhī pour noter le sanskrit et même des langues non indo-aryennes, c'était essentiellement l'écriture qui notait la gāndhārī, un dialecte moyen-indien parlé au Nord-Ouest du monde indien, correspondant en gros au NordOuest du Pakistan actuel.

L'émergence d'une collection importante de manuscrits au début des années 1990, qui fut acquise par la British Library (Londres), a ouvert une série de nouvelles trouvailles, en sorte qu'à présent on connaît plus d'une centaine de manuscrits écrits en kharoșthī, dans leur majorité de contenu bouddhique. Parmi ces lots de manuscrits, une collection d'une importance significative est l'objet d'un projet de recherche de l'université de Berlin, Freie Universität, le « Bajaur Collection Project». Cette documentation a servi de base aux trois premières conférences, qui ont été consacrées aux différents aspects de la littérature bouddhique et de la civilisation indienne dans l'ancien 
Gandhāra. L'accent a été placé sur le rôle historique et culturel de cette région dans la transmission du bouddhisme depuis l'Inde jusqu'en Asie centrale et en Chine.

La dernière conférence a traité d'une autre découverte spectaculaire dans le domaine de l'épigraphie indienne. En 2000 des spéléologues belges ont découvert dans l'une des gigantesques grottes de l'île de Socotra (proche du Yémen) un grand nombre d'inscriptions, parmi lesquelles plus de 180 graffiti en écriture $b r a \bar{h} m \bar{\imath}$, l'autre type d'écriture du monde indien, gravés par des commerçants indiens. Cette trouvaille élargit de manière considérable le champ de l'épigraphie indienne et jette une lumière nouvelle sur les activités de commerce maritime de l'Inde dans les premiers siècles de notre ère.

\section{Les manuscrits en gāndhārī et la culture bouddhique au Gandhāra}

Cette conférence a présenté les matériaux récemment découverts et leurs caractéristiques; elle a aussi donné l'état présent des recherches. Une attention particulière a été portée à la collection de Bajaur, que le maître de conférences invité étudie luimême depuis 2005. En même temps, l'intention était de traiter des problèmes principaux qui concernent la littérature ancienne du Gandhāra dans son ensemble. La discussion n'a pas seulement porté sur l'arrière-plan historique et géographique de la civilisation bouddhique au Gandhāra; elle a aussi fait référence aux autres sites principaux de la documentation manuscrite du bouddhisme ancien, comme par exemple Gilgit et les oasis d'Asie centrale.

La conférence a traité des genres des textes qui ont été identifiés jusqu'à présent dans les collections connues de manuscrits en gāndhārī. On a notamment posé la question du rapport entre la multitude de textes différents déjà connus et l'existence supposée d'un canon de littérature bouddhiste en gāndhārī. Cette question est étroitement liée à celle de l'affiliation sectaire de ces textes. Bien qu'il y ait une certaine présomption en faveur d'un lien avec l'école Dharmaguptaka, un grand nombre de problèmes restent à résoudre avant que l'on puisse proposer une attribution précise des textes en gāndhārī. La conférence a essayé de mettre en exergue les points suivants : 1) Que savons-nous de la forme des diverses compilations canoniques à l'époque de la composition des textes en gāndhārī? 2) Que savons-nous de l'activité des sectes (ou écoles) bouddhiques au Gandhāra? 3) Sur quelle base pouvons-nous attribuer clairement certains textes en gāndhārī à une école particulière ? Ce dernier point a été discuté de façon approfondie à partir des textes de Vinaya de la collection de Bajaur, pour lesquels on peut trouver de nombreux parallèles dans d'autres traditions bouddhiques.

\section{L'essor du Mahāyāna et l'ancienne littérature du Mahāyāna en gāndhārī}

Un nombre considérable de traductions chinoises anciennes de traités (sūtra) du Mahāyāna présentent des traces d'une rédaction originale en gāndhārī, et non pas en sanskrit. Il a donc été suggéré depuis longtemps qu'il a existé un corpus important de littérature du Mahāyāna en gāndhārī. Cela restait une hypothèse avant les découvertes les plus récentes. 
Parmi les textes de Mahāyāna en gāndhārī qui ont été récemment mis au jour figure un sūtra sur le Buddha Akṣobhya, lequel fait partie de la collection de Bajaur. D'après sa taille, il s'agit du plus important texte connu en gāndhārī après le Dharmapada de Khotan : il comprend plus de 640 lignes sur les deux faces d'un rouleau mesurant plus de $220 \mathrm{~cm}$. La conférence a donné un aperçu de la recherche en cours sur ce texte fondamental et a exposé les résultats provisoires, en relation avec les différents genres de la littérature ancienne du Mahāyāna, comme par exemple, la littérature de Prajñāpāramitā et de « Terre Pure ». On a insisté sur le rôle d'Akṣobhya et de son paradis Abhirati dans la structure de ce texte. La dernière partie de la conférence a discuté la portée de ces découvertes au regard des théories en présence sur le milieu social et culturel qui a vu l'émergence du bouddhisme Mahāyāna.

\section{Le déclin de la gāndhārī et de la kharoșthī, corollaire de la sanskritisation de la culture indienne}

Au Gandhāra proprement dit, l'usage de la gāndhārī comme langue littéraire et administrative, associé à celui de la kharoșţhī qui notait cette langue, est en voie de disparition à la fin $\mathrm{du}_{\mathrm{III}}^{\mathrm{e}}$ siècle de notre ère. Jusqu'à présent, les raisons alléguées pour ce déclin sont plutôt contradictoires. Tandis que certains savants invoquent des causes politiques, telles que la fin de la domination kouchane (Kușanna), d'autres affirment que le déclin de la culture bouddhique est responsable de l'obsolescence de la kharoṣthī. Dans cette conférence, l'hypothèse de travail a été la suivante : c'est le processus panindien de sanskritisation qui a causé l'abandon progressif de la langue gāndhārī et de l'écriture kharoșthī. Cette hypothèse a été testée à partir d'une révision de toute la documentation disponible sur la phase finale de l'usage de la kharoșțī au Gandhāra et dans les régions adjacentes (Bactriane, Asie centrale). Sur la base des données que fournit la collection de Bajaur, on a montré que c'est la structure phonétique du système graphique de la kharoșțhī qui a favorisé son remplacement au cours du processus de sanskritisation. En effet, alors que cette écriture était tout à fait adéquate pour fixer les différents parlers de l'aire linguistique de la gāndhārī, elle se révélait inadaptée à la notation de textes sanskrits. On a aussi attiré l'attention sur les conditions particulières dans lesquelles l'écriture kharoșthī a survécu bien après le III $^{\mathrm{e}}$ siècle dans des régions d'Asie centrale périphériques par rapport au Gandhāra.

\section{Socotra après le "Périple » : les activités de commerce intercontinental des marchands indiens au cours des premiers siècles de notre ère}

En 2000 des spéléologues belges ont découvert un grand nombre d'inscriptions indiennes au cours de l'exploration de gigantesques cavernes naturelles sur l'île de Socotra. Grâce aux membres du « Sokotra Karst Project », le conférencier a pu participer à la huitième expédition de cette équipe, du 29 décembre 2005 au 15 janvier 2006. Une nouvelle exploration a permis de découvrir dans une caverne une série d'inscriptions indiennes jusqu'à présent inédites, parmi lesquelles on trouve des graffiti en brāhmī qui mentionnent l'origine des visiteurs et même une courte inscription en kharoșțī. Il a été possible d'identifier au total plus de 180 inscriptions indiennes. 
Le corpus de Socotra est un témoignage unique sur les activités indiennes de commerce maritime dans la mer d'Arabie (mer d'Oman). En dehors d'un petit nombre de textes en brāhmī sur des tessons provenant de Bérénice (port sur la mer Rouge, au Sud de l'Égypte), il constitue le point le plus occidental de l'expansion d'une écriture indienne. De plus, il confirme l'authenticité de sources anciennes comme le Périple de la mer Érythrée, qui signalait la présence d'Indiens sur cette île dès le premier siècle de notre ère.

La conférence a présenté les résultats de l'analyse épigraphique et historique du corpus. Elle a mis cette documentation en relation avec les activités de commerce par mer entre l'Inde et Rome, telles qu'elles sont connues par des sources littéraires et archéologiques. L'analyse épigraphique inclut aussi du matériel connu par le souscontinent indien, en particulier les nombreux graffiti découverts le long de la Karakorum Highway au Nord du Pakistan actuel. 